\title{
Radiation recall pneumonitis triggered by an immune checkpoint inhibitor following re-irradiation in a lung cancer patient: a case report
}

\author{
Xianghua $\mathrm{Ye}^{1+}{ }^{1}$, Jinsong Yang ${ }^{1 \dagger}$, Justin Stebbing ${ }^{2}$ and Ling Peng ${ }^{3^{*}}$ (i)
}

\begin{abstract}
Background: Radiation recall pneumonitis (RRP) is unpredictable but associated with severe radiation damage in previously irradiated fields. Chemotherapy and targeted drugs have been reported to contribute to RRP. Here we report a case of a patient with non-small cell lung cancer (NSCLC) who developed RRP following administration of immune checkpoint inhibitor (ICI) 18 months after the end of re-irradiation.

Case presentation: A 69-year-old man received adjuvant chemoradiotherapy post-operatively. He underwent thoracic re-irradiation for oligometastatic NSCLC. On second recurrence, pembrolizumab combined with nab-paclitaxel were administered. After six months, he developed symptoms of persistent cough and dyspnea, with consistent pneumonitis on CT images. The clinical time frame and significant radiographic evidence raised suspicion for RRP. Symptoms resolved after steroids.
\end{abstract}

Conclusions: RRP is a rare occurrence. Patients undergoing immunotherapy after prior irradiation may be at increased risk of this rare radiation pneumonitis.

Keywords: Radiation recall pneumonitis, Radiotherapy, Immunotherapy, NSCLC

\section{Background}

Pneumonitis may be a potential result of both thoracic radiation and ICI, particularly treatment with programmed death-1 (PD-1) or programmed death ligand-1 (PDL-1) inhibitors [1]. The differential diagnosis between radiation pneumonitis and checkpoint inhibitor-induced pneumonitis (CIP) is of importance for patients receiving both treatments. RRP is described as a delayed inflammatory reaction occurring within irradiated tissues. RRP

*Correspondence: drpengling@hotmail.com

${ }^{\dagger}$ Xianghua Ye and Jinsong Yang have contributed equally to this work

${ }^{3}$ Cancer Center, Department of Pulmonary and Critical Care Medicine, Zhejiang Provincial People's Hospital, Affiliated People's Hospital, Hangzhou Medical College, Hangzhou 310014, Zhejiang Province, China Full list of author information is available at the end of the article is rare but is reported to be triggered by chemotherapy agents such as anthracyclines, taxanes, and gemcitabine $[2,3]$, and targeted therapies such as erlotinib, osimertinib and vemurafenib [4, 5]. During the COVID-19 era, RRP has been reported with vaccination [6]. RRP induced by ICI has been reported, but it is uncommon in patients receiving ICI with a pre-irradiation history.

Here, we described a male patient diagnosed with primary lung squamous carcinoma who received surgery. Post-operative chemotherapy and radiotherapy were given as adjuvant therapy. On recurrence of lymph nodes 3 years post-surgery, re-irradiation was administered to mediastinal lymph nodes and chemotherapy was given. On second recurrence 4 years after surgery, systemic therapy with pembolizumab and nab-paclitaxel was 
given. After 6 months following initiation of pembrolizumab and nab-paclitaxel, he experienced RRP occurring 18 months after the end of re-irradiation. Steroid therapy resulted in alleviation of symptoms in 3 days and radiological improvement after one month.

\section{Case presentation}

A 64-year-old Chinese male smoker referred to The First Affiliated Hospital of Zhejiang University in August 2016 due to a one-month history of cough. His chest CT scan revealed a $3 \mathrm{~cm} \times 2.5 \mathrm{~cm}$ mass in the left upper lobe and mediastinal lymph node enlargement (Fig. 1A). A PETCT indicated the mass with elevated SUV and ipsilateral mediastinal lymph nodes (Fig. 1B). Past and family histories were unremarkable. Due to the staging of the disease, he was considered to be a candidate for curative surgical excision. On August 11th, 2016, this patient underwent lobectomy of left upper lobe and mediastinal lymph node dissection. Histology returned with a poorly differentiated squamous carcinoma with involvement of elastic membrane and vascular tumor embolus. The surgical margins were negative. Mediastinal lymph nodes of station 7 were metastatic with pathological stating of pT2N2M0, IIIA. Sequential chemoradiotherapy with gemcitabine/cisplatin and radiotherapy 50 Gy in 25 fractions was started 1 month after the lobectomy using Intensity-modulated radiation therapy (IMRT). Clinical target volume (CTV) includes tumor area, 4L, 5, 7 and 10L lymph node stations (Fig. 1C, D). After adjuvant chemoradiotherapy, this patient underwent routine follow-up regularly. No radiation pneumonitis was observed.

On May 2019, a chest CT scan revealed lymph node enlargement. A second PET-CT scan confirmed elevated SUV uptake of 2L, 7 stations and right hilar lymph nodes (Fig. 2A, C, D). The patient was seen in multidisciplinary follow up, and a recommendation was made for first-line chemotherapy followed by re-irradiation, which would be optimal for local-recurrence of the disease. Nabpaclitaxel with carboplatin were given for four cycles. Then, re-irradiation was given with $54 \mathrm{~Gy} / 27 \mathrm{~F}$ including $2 \mathrm{R}, 7,10 \mathrm{R}$ lymph node stations from September 17 th to November 14th 2019, using three-dimensional conformal radiation therapy (3D CRT) (Fig. 2E, F). The lymph nodes are shown on the follow-up CT scan one month after the end of re-irradiation (Fig. 2G, H). He tolerated well with
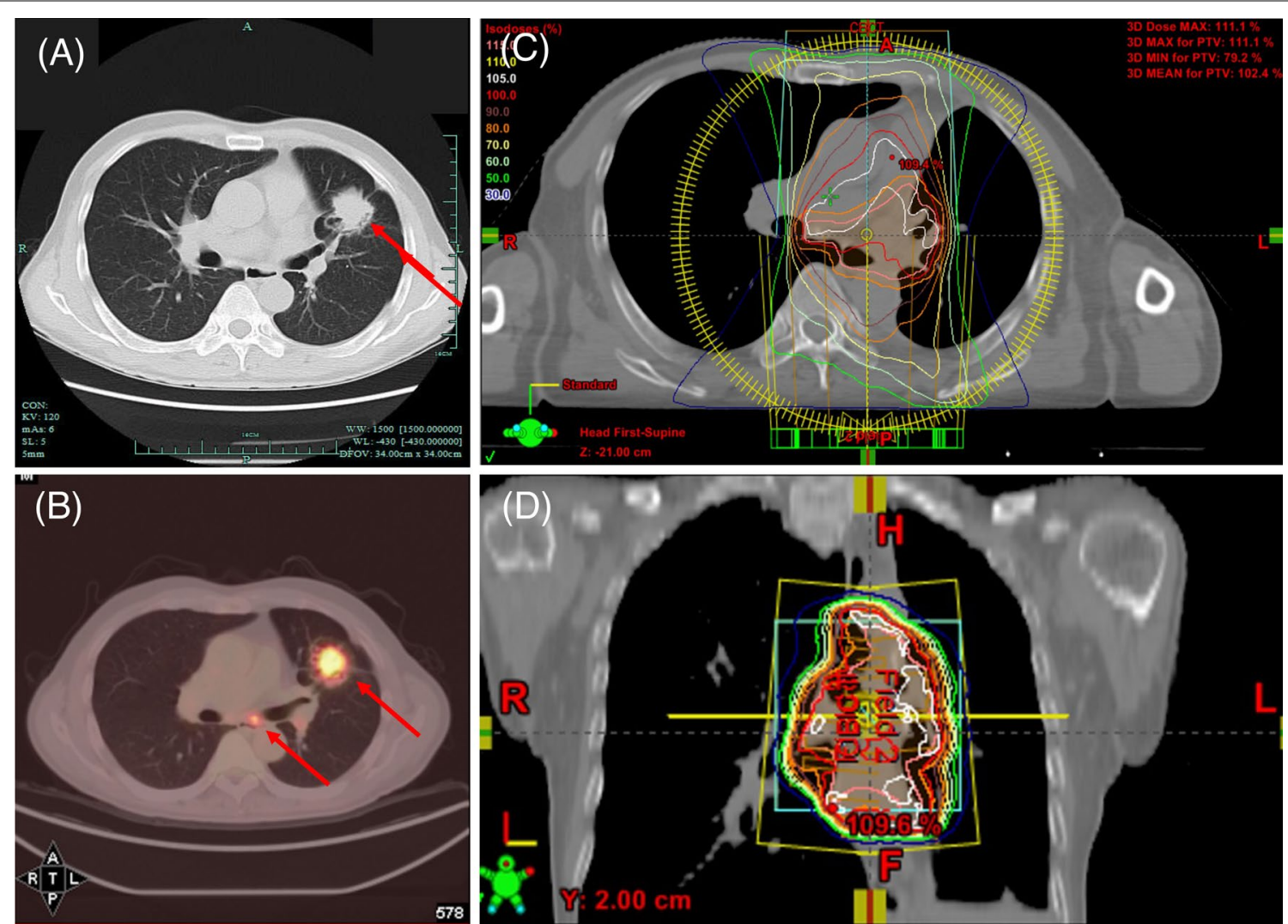

Fig. 1 A CT scan of lung window captured on August 6th, 2016; B PET-CT captured on August 8th, 2016; C, D Radiation dosimetry post-operation. Significant abnormal findings noted (arrow) 

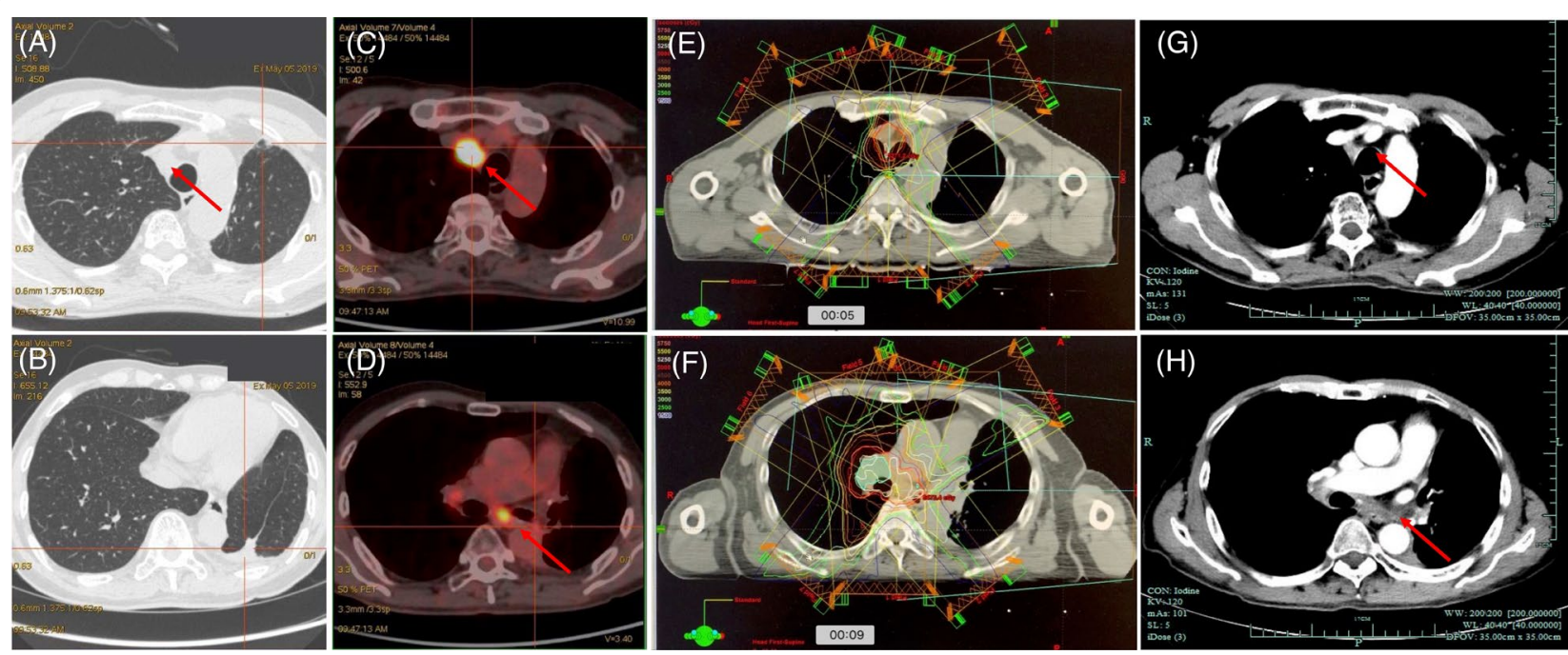

Fig. 2 A-D PET-CT scan capture on May 5th, 2019. E, F Radiation dosimetry of re-irradiation; G, H CT lung and contrast window capture on Octobe 29th, 2019 (after re-irradiation). Significant abnormal findings noted (arrow)

radiotherapy and there were no symptoms. CT findings revealed no signs of radiation pneumonitis.

On November 2020, a CT scan revealed enlargement of lymph nodes compared with the best response to reirradiation captured on November 2019 (Fig. 3A-D). The diagnosis of second recurrence was made. After a second multidisciplinary discussion, pembrolizumab $200 \mathrm{mg}$ plus nab-paclitaxel $200 \mathrm{mg} / \mathrm{m}^{2}$ every 3 weeks were given in combination with stable disease.

On April 20th, 2021, he complained of persistent, nonproductive cough and dyspnea, with no fever. CT images on May 1st, 2021 indicated patchy consolidation located in the previously re-irradiated area (Fig. 3E, F). Bronchoscopy revealed no abnormalities (Fig. 3G, H). No evidence of infection and cancer cells was found from bronchoalveolar lavage fluid (BALF). Blood tests were negative for tumor marker. Cytokine levels were assessed including interleukin-2 (IL-2), IL-1, IL-6, IL-8, IL-17, interferon gamma (IFN- $\gamma$ ); all of which were within normal range. Steroids were given using $40 \mathrm{mg}$ methylprednisolone intravenously q12h for 3 days. Concurrent moxifloxacin was given due to the potential of overlapping presentation and infection. The symptoms quickly resolved in 3 days. Steroids were tapered gradually in 6 weeks. A
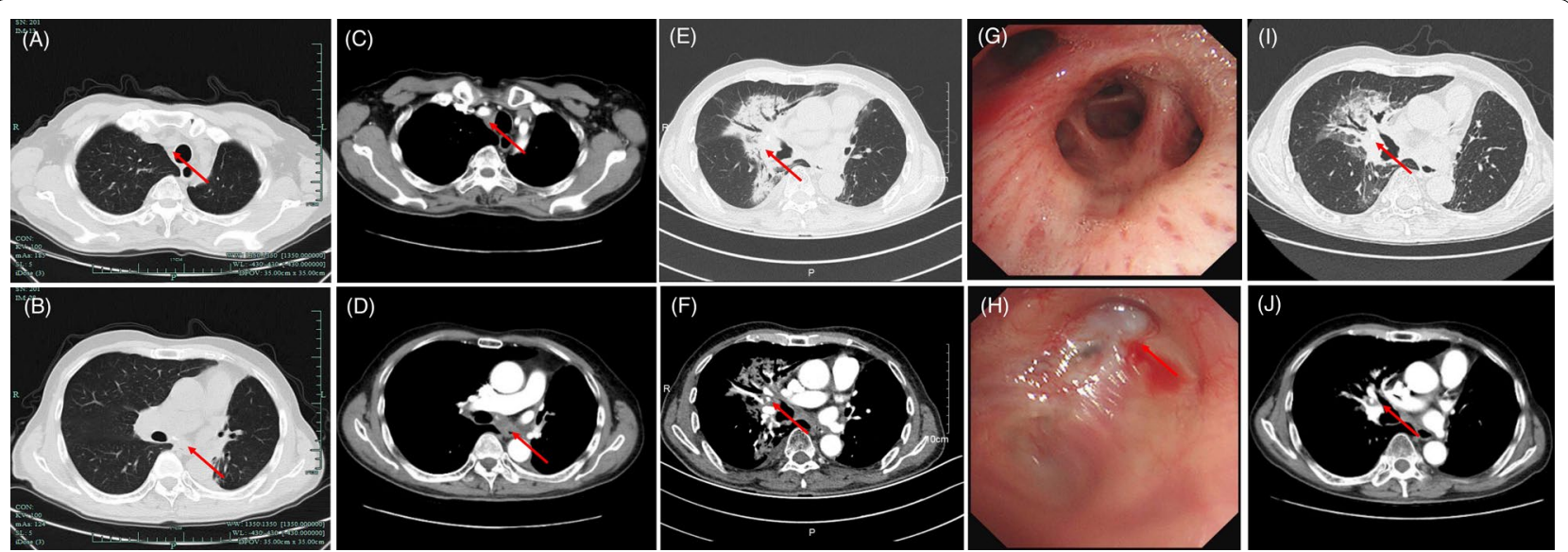

Fig. 3 CT lung window (A, B) and contrast window (C, D) before pembrolizumab plus nab-paclitaxel, captured on November 5th, 2020; E, F CT lung and contrast window on May 1st, 2021; G The mucosa of right middle bronchus: is normal and the bronchus is clear. $\mathbf{H}$ The mucosa of the left upper lobe is post-surgery appearance and the bronchus is clear. I, J CT lung and contrast window captured on June 5th, 2021 (one month after steroids). Significant abnormal findings noted (arrow) 
repeated CT scan on June 5th, 2021 revealed significant improvement of RRP (Fig. 3I, J). The timeline of his diagnosis, treatment and onset of RRP are shown in Fig. 4. At the time of writing this report, the patient is still on active follow-up.

\section{Discussion and conclusions}

Radiation is a potent inflammation inducer. Radiation induces immunogenic cell death, which results in release of damage-associated molecular pattern (DAMP) markers [7]. Being a generator of danger signals, radiation also notifies the immune system to potential damage. Radiation has immunomodulatory effects, and the reaction of the immune system is crucial to the clinical response to radiation therapy. Inflammatory reactions are orchestrated by innate immune cells and the adaptive immune system.

With the wide application of ICIs, it is rational to explore the capacity of radiation to enhance ICI efficacy and to investigate whether combination therapy could bring benefit to patients with cancer. The overlapping pulmonary toxicity induced by thoracic RT and ICI is an important issue. Concurrent treatment with ICI and conventional therapies may also result in higher rates of pneumonitis. The safety of combination of radiotherapy and immunotherapy has been evaluated in a variety of clinical trials and real-world data. In a phase III randomized trial investigating durvalumab after concurrent chemoradiotherapy (CCRT) in stage III NSCLC, the pneumonitis rate was reported as $34 \%$, compared to $25 \%$ in placebo arm [8]. Pneumonitis was the most frequent adverse event $(\mathrm{AE})$ leading to drug discontinuation $(4.8 \%$ in the durvalumab group and in $2.6 \%$ of those in the placebo group). In a real-world setting, NSCLC patients previously treated with radiotherapy had a higher risk for CIP [9]. Grade $\geq 2$ CIP is an independent prognostic factor for shorter survival in NSCLC patients. On the other hand, ICI could also increase the risk for radiationinduced pneumonitis.
The differentiation of RRP and CIP is challenging in clinical setting. The clinical presentation of RRP was different from common radiation pneumonitis and RRP induced by cytotoxic/targeted drugs. RRP and CIP could both present with similar images on CT scans, and they usually respond well to steroid therapy. While the images of ICI-induced RRP represent the target volume of irradiated fields and are more focal than traditional CIP, this helps clinicians to reach a diagnosis. Radiological features of ICI-induced RRP include ground-glass or consolidative opacities confined to prior radiation field [10]. The most common radiographic patten of RRP is cryptogenic organizing pneumonia (COP) [11]. Artificial intelligence and radiomics have also emerged as promising tools to interrogate cancer images before, during and after treatment [12].

RRP is an unpredictable event, whose mechanism is not fully understood. There are some explanations postulated [11] including changes in the function of stem cells in the irradiated field versus idiosyncratic drug hypersensitivity reactions [13]. Other mechanisms as drug hypersensitivity, vascular injury or inflammatory cascades have also been suggested [14]. While the exact mechanism of RRP induced by ICI is somewhat different from conventional chemotherapy or targeted drugs, the signaling pathways involved in immunotherapy-induced RRP are thought to include cGMP-AMP synthase (cGAS)stimulator of interferon genes (STING), nuclear factor kappa-light-chain-enhancer of activated B cells (NF-kB), reactive oxygen species/reactive nitrogen species (ROS/ RNS), extracellular regulated protein kinases (Erk) and phosphatidylinositol 3-kinase (PI3K) [11].

RRP manifests as acute inflammation lymphocytes infiltration, which can occur weeks, months, or even years after radiotherapy in response to treatment. RRP is a delayed radiation-induced lung toxicity, with long interval between the administration of ICI and the onset of RRP being observed. With conventional agents, the median time of onset of RRP after the end of radiation therapy is 95 days for chemotherapy [2] and 124 days

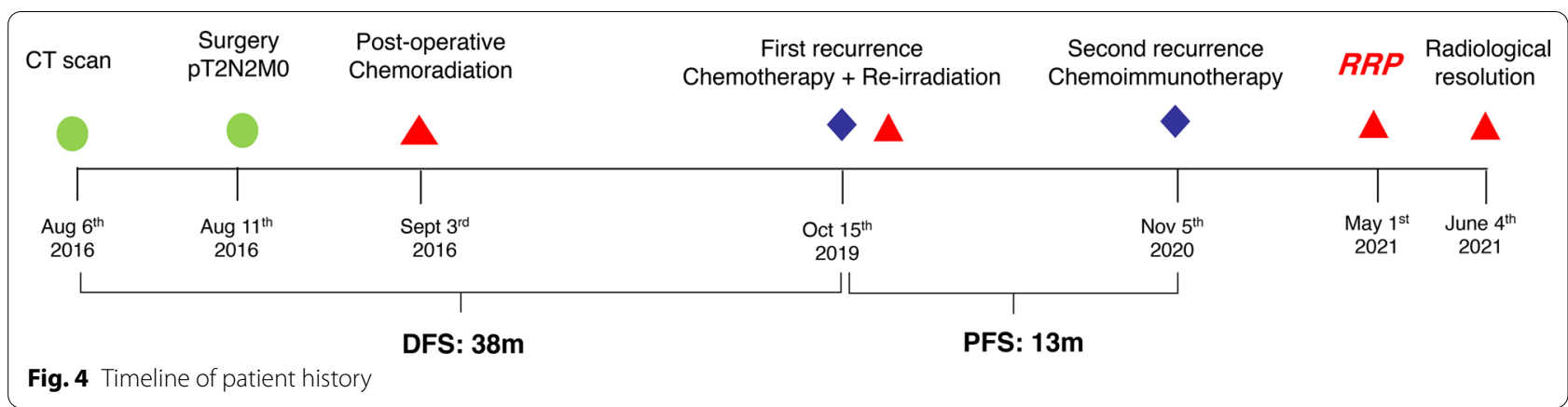


for targeted therapy [15], although onset of 2 years after radiation therapy has been reported with ICIs [16]. In our case, the onset of symptoms developed 18 months after the end of re-irradiation. The published cases of ICI-induced RRP are listed in Table 1, and the time interval of RRP to radiotherapy varies widely.

The risk factor for RRP have not been determined, but several factors might contribute [17]: (i) Radiotherapy. The dose, fractionation, target volume, radiation technique could affect the toxicity of radiation [11]. As radiation pneumonitis is closely related to dosimetric factors, close monitoring and attention shall be given to patients receiving combination therapy. In our case report, this patient received operation upfront, followed by chemoradiation $[18,19]$. In patients receiving re-irradiation, dose-volume variables from re-RT plan and factors from initial-RT are predictive factors for severe radiation pneumonitis [20]. (ii) Drugs used. Although chemotherapy, targeted drugs and ICIs can all trigger RRP, the ICI-induced RRP is different, in terms of a long interval and duration of response. In our case, this patient also received nab-paclitaxel with pembrolizumab. As a taxane is one of the drugs potentially contributing to RRP, the role of nab-paclitaxel in the development of RRP cannot be ruled out. However, as the dose and density of nabpaclitaxel was standard, we suspect the main drug in responsible for RRP here was pembrolizumab. Although previously reported, it was concluded that the pneumonitis was RRP which is a rare inflammatory reaction in the previously irradiated lung field was due to administration of this ICI. The incidences of CIP induced by anti-PD-1 antibody are higher compared to anto-PD-L1 antibody, especially grade $3-4$ pneumonitis [21]. Therefore, RRP is more likely to be triggered by PD-1 inhibitor than PD-L1 inhibitor. (iii) Tumor location, molecular features and type. Whether PD-L1 status will affect the toxicity of radiation in combination with ICI remains unknown. In this patient, PD-L1 status was undermined due to the fact that his tumor sample was in 2016, when the diagnostic kit was not available in China and the achieved sample was not suitable for PD-L1 test after 5 years. Other factors as TMB (tumor mutation burden), baseline immune status, MSI status, and other peripheral blood biomarkers might also influence the onset of RRP.

Interestingly, a systematic review found that the occurrence of immune-related adverse events (irAEs) was significantly associated with a better ICI efficacy in patients with cancer, particularly endocrine, dermatological, and low-grade irAEs [22]. RRP may reflect a beneficial immune activation and constitute a predictive biomarker for long-term efficacy [23].

In this report, we describe a NSCLC patient of RRP triggered by pembrolizumab 18 months after the end of re-irradiation. RRP induced by ICIs is a unique pattern of radiation-related toxicity. Clinicians should be aware of the pulmonary toxicity even years after radiation, especially in the era of immunotherapy.

\section{Abbreviations}

RRP: Radiation recall pneumonitis; NSCLC: Non-small cell lung cancer; ICl: Immune checkpoint inhibitor; PD-1: Programmed death-1; CIP: Checkpoint inhibitor-induced pneumonitis; CTV: Clinical target volume; 3D-CRT: Threedimensional conformal radiation therapy; IFN- $\gamma$ : Interferon gamma; DAMP: Damage-associated molecular pattern; CCRT: Concurrent chemoradiotherapy; AE: Adverse event; COP: Cryptogenic organizing pneumonia; CGAS: CGMPAMP synthase; STING: CGAS-stimulator of interferon genes; NF-kB: Nuclear factor kappa-light-chain-enhancer of activated B cells; ROS: Reactive oxygen species; RNS: Reactive nitrogen species; Erk: Extracellular regulated protein kinases; PI3K: Phosphatidylinositol 3-kinase; irAE: Immune-related adverse events.

Table 1 RRP Cases reported

\begin{tabular}{lllllllll}
\hline No & Author & Year & Country & Sex & Age & Malignancy & ICI & Onset time from RT \\
\hline 1 & Current paper & 2021 & China & Male & 69 & NSCLC & Pembrolizumab & 18 months \\
2 & Riviere [24] & 2021 & United States & Male & 64 & NSCLC & Nivolumab & 4.5 years \\
& & & & Male & 67 & Bladder & Ipilimumab-Pembrolizumab & 6 months \\
& & & & Male & 52 & SCLC & Nivolumab-Ipililumab & 7 months \\
3 & De Giglio [25] & 2021 & Italy & Male & 53 & RCC & Nivolumab & 10 months \\
4 & Itamura [26] & 2020 & Japan & NR & NR & NSCLC & Pembrolizumab & 7 months \\
5 & Chen [27] & 2020 & China & Male & 64 & NSCLC & Camrelizumab & 2 years \\
6 & Deutsch [23] & 2020 & France & NR & NR & NSCLC & Nivolumab & NR \\
7 & Wang [28] & 2020 & China & Male & 52 & SCLC & Pembrolizumab & 6 months \\
8 & McGovern [29] & 2019 & United States & Male & 82 & NSCLC & Pembrolizumab & 14 months \\
9 & Nakamura [30] & 2019 & Japan & Female & 69 & RCC & Nivolumab & 9 months \\
10 & Shibaki [16] & 2017 & Japan & Male & 68 & NSCLC & Nivolumab & 2 years \\
& & & Male & 55 & NSCLC & Nivolumab & 6 months
\end{tabular}

$R T$ radiotherapy; NSCLC non-small cell lung cancer; SCLC small cell lung cancer; $R C C$ renal cell carcinoma; NR not reported 


\section{Acknowledgements \\ None.}

\section{Authors' contributions}

$X Y$ and $L P$ wrote the manuscript. $X Y, L P$, JS and JY revised the manuscript and provided guidance. $X Y, L P$ and $J Y$ took care of the patient. All authors listed have made a substantial, direct and intellectual contribution to the work. All authors read and approved the final manuscript.

\section{Funding}

This study was supported by a grant from Medical Science Research Foundation of Health Bureau of Zhejiang Province (Grant Number: 2022KY545) and a grant from the Administration of Traditional Chinese Medicine of Zhejiang Province (Grant Number: 2022ZA021). The funding bodies played no role in the design of the study and collection, analysis, and interpretation of data and in writing the manuscript.

\section{Availability of data and materials}

The datasets used and/or analysed during the current study are available from the corresponding author on reasonable request.

\section{Declarations}

\section{Ethics approval and consent to participate}

Not applicable.

\section{Consent for publication}

Written informed consent was obtained from the case patient for publication of this report and images. A copy of the written consent is available for review by the Editor of this journal.

\section{Competing interests}

Professor Justin Stebbing is the Editor-in-Chief of Oncogene. JS' conflicts can be found at: https://www.nature.com/onc/editors. None are relevant here. No other author declares a conflict.

\section{Author details}

${ }^{1}$ Department of Radiotherapy, The First Affiliated Hospital, School of Medicine, Zhejiang University, Hangzhou, Zhejiang Province, China. '2 Division of Cancer, Department of Surgery and Cancer, Imperial College London, London, UK. ${ }^{3}$ Cancer Center, Department of Pulmonary and Critical Care Medicine, Zhejiang Provincial People's Hospital, Affiliated People's Hospital, Hangzhou Medical College, Hangzhou 310014, Zhejiang Province, China.

Received: 12 November 2021 Accepted: 29 January 2022

Published online: 05 February 2022

\section{References}

1. Schoenfeld JD, Nishino M, Severgnini M, Manos M, Mak RH, Hodi FS. Pneumonitis resulting from radiation and immune checkpoint blockade illustrates characteristic clinical, radiologic and circulating biomarker features. J Immunother Cancer. 2019;7(1):112.

2. Ding X, Ji W, Li J, Zhang X, Wang L. Radiation recall pneumonitis induced by chemotherapy after thoracic radiotherapy for lung cancer. Radiat Oncol. 2011:6:24.

3. Tomiguchi M, Yamamoto Y, Hayashi M, Yamamoto-Ibusuki M, Murakami $\mathrm{K}$, Iwase H. Docetaxel and cyclophosphamide chemotherapy induced radiation recall phenomenon in a postoperative breast cancer patient: a case report. Int Cancer Conf J. 2016;5(4):202-5.

4. Onal C, Abali H, Koc Z, Kara S. Radiation recall pneumonitis caused by erlotinib after palliative definitive radiotherapy. Onkologie. 2012:35(4):191-4

5. Forschner A, Zips D, Schraml C, Rocken M, lordanou E, Leiter U, Weide B, Garbe C, Meier F. Radiation recall dermatitis and radiation pneumonitis during treatment with vemurafenib. Melanoma Res. 2014:24(5):512-6.
6. Soyfer V, Gutfeld O, Shamai S, Schlocker A, Merimsky O. COVID-19 vaccine-induced radiation recall phenomenon. Int J Radiat Oncol Biol Phys 2021.

7. Mavragani IV, Laskaratou DA, Frey B, Candeias SM, Gaipl US, Lumniczky K, Georgakilas AG. Key mechanisms involved in ionizing radiation-induced systemic effects. A current review. Toxicol Res (Camb). 2016;5(1):12-33.

8. Antonia SJ, Villegas A, Daniel D, Vicente D, Murakami S, Hui R, Yokoi T, Chiappori A, Lee $\mathrm{KH}$, de Wit M, et al. Durvalumab after chemoradiotherapy in stage III non-small-cell lung cancer. N Engl J Med. 2017:377(20):1919-29.

9. Barron F, Sanchez R, Arroyo-Hernandez M, Blanco C, Zatarain-Barron ZL, Catalan R, Ramos-Ramirez M, Cardona AF, Flores-Estrada D, Arrieta O. Risk of developing checkpoint immune pneumonitis and its effect on overall survival in non-small cell lung cancer patients previously treated with radiotherapy. Front Oncol. 2020;10:570233.

10. Kalisz KR, Ramaiya NH, Laukamp KR, Gupta A. Immune checkpoint inhibitor therapy-related pneumonitis: patterns and management. Radiographics. 2019;39(7):1923-37.

11. Teng F, Li M, Yu J. Radiation recall pneumonitis induced by PD-1/ PD-L1 blockades: mechanisms and therapeutic implications. BMC Med. 2020;18(1):275

12. Tunali I, Gillies RJ, Schabath MB. Application of radiomics and artificial intelligence for lung cancer precision medicine. Cold Spring Harb Perspect Med 2021.

13. Burris HA 3rd, Hurtig J. Radiation recall with anticancer agents. Oncologist. 2010;15(11):1227-37.

14. Levy A, Hollebecque A, Bourgier C, Loriot Y, Guigay J, Robert C, Delaloge S, Bahleda R, Massard C, Soria JC, et al. Targeted therapy-induced radiation recall. Eur J Cancer. 2013;49(7):1662-8.

15. Chiang $\mathrm{CL}$, Chen YW, Wu MH, Huang HC, Tsai CM, Chiu CH. Radiation recall pneumonitis induced by epidermal growth factor receptor-tyrosine kinase inhibitor in patients with advanced nonsmall-cell lung cancer. J Chin Med Assoc. 2016;79(5):248-55.

16. Shibaki R, Akamatsu H, Fujimoto M, Koh Y, Yamamoto N. Nivolumab induced radiation recall pneumonitis after two years of radiotherapy. Ann Oncol. 2017:28(6):1404-5.

17. Cousin F, Desir C, Ben Mustapha S, Mievis C, Coucke P, Hustinx R. Incidence, risk factors, and $C T$ characteristics of radiation recall pneumonitis induced by immune checkpoint inhibitor in lung cancer. Radiother Oncol. 2021;157:47-55.

18. Spoelstra FO, Senan S, Le Pechoux C, Ishikura S, Casas F, Ball D, Price A, De Ruysscher D, van Sornsen de Koste JR. Lung adjuvant radiotherapy trial investigators G: VARIATIONS in target volume definition for postoperative radiotherapy in stage III non-small-cell lung cancer: analysis of an international contouring study. Int J Radiat Oncol Biol Phys. 2010;76(4):1106-13.

19. Gopal RS, Dubey S, Rosenzweig KE, Chang JY, Decker R, Gewanter RM, Kong FM, Lally BE, Langer CJ, Lee HK, et al. ACR Appropriateness criteria(R) on induction and adjuvant therapy for stage N2 non-small-cell lung cancer: expert panel on radiation oncology-lung. Int J Radiat Oncol Biol Phys. 2010;78(4):969-74.

20. Ren C, Ji T, Liu T, Dang J, Li G. The risk and predictors for severe radiation pneumonitis in lung cancer patients treated with thoracic reirradiation. Radiat Oncol. 2018;13(1):69.

21. Khunger M, Rakshit S, Pasupuleti V, Hernandez AV, Mazzone P, Stevenson J, Pennell NA, Velcheti V. Incidence of pneumonitis with use of programmed death 1 and programmed death-ligand 1 inhibitors in nonsmall cell lung cancer: a systematic review and meta-analysis of trials. Chest. 2017;152(2):271-81.

22. Zhou $X$, Yao Z, Yang H, Liang N, Zhang X, Zhang F. Are immune-related adverse events associated with the efficacy of immune checkpoint inhibitors in patients with cancer? A systematic review and meta-analysis. BMC Med. 2020;18(1):87.

23. Deutsch E, Besse B, Le Pavec J, Le Pechoux C, Botticella A, Ammari S, Even C, Robert C, Levy A. Can radiation-recall predict long lasting response to immune checkpoint inhibitors? Radiother Oncol. 2021;154:125-7.

24. Riviere P, Sumner W, Cornell M, Sandhu A, Murphy JD, Hattangadi-Gluth J, Bruggeman A, Kim SS, Randall JM, Sharabi AB. Radiation recall pneumonitis after treatment with checkpoint blockade immunotherapy: a case series and review of literature. Front Oncol. 2021;11:662954

25. De Giglio A, Scorsetti M, Franceschini D, Massari F, Ardizzoni A. Bilateral radiation recall pneumonitis during immunotherapy for an advanced 
renal cell carcinoma: A challenging case enhances the need for a multidisciplinary approach. Eur J Cancer. 2021;143:75-7.

26. Itamura H, Ohguri T, Yahara K, Nakahara S, Kakinouchi S, Morisaki T, Yatera K, Tanaka F, Korogi Y. Pembrolizumab-induced radiation recall pneumonitis after the resolution of typical asymptomatic radiation pneumonitis. J UOEH. 2020;42(3):261-6.

27. Chen Y, Huang Z, Xing L, Meng X, Yu J. Radiation recall pneumonitis induced by anti-PD-1 blockade: a case report and review of the literature. Front Oncol. 2020;10:561.

28. Wang YY, Tian XC, Zhu L, Bai XH, Zhao R. Concomitant radiation recall dermatitis and radiation recall pneumonitis induced by pembrolizumab. J Thorac Oncol. 2020;15(10):e160-2.

29. McGovern K, Ghaly M, Esposito M, Barnaby K, Seetharamu N. Radiation recall pneumonitis in the setting of immunotherapy and radiation: a focused review. Future Sci OA. 2019;5(5):FSO378.

30. Nakamura K, Okubo K, Takahashi T, Mitsumori K, Ishigaki T, Ohnishi H. Radiation recall pneumonitis induced by nivolumab in a patient with renal cell carcinoma. IJU Case Rep. 2019;2(1):30-3.

\section{Publisher's Note}

Springer Nature remains neutral with regard to jurisdictional claims in published maps and institutional affiliations.

- fast, convenient online submission

- thorough peer review by experienced researchers in your field

- rapid publication on acceptance

- support for research data, including large and complex data types

- gold Open Access which fosters wider collaboration and increased citations

- maximum visibility for your research: over 100M website views per year

At BMC, research is always in progress.

Learn more biomedcentral.com/submissions 